\title{
The Acoustic Method of Depth Sounding for Navigation Purposes. ${ }^{1}$
}

$\mathrm{O}^{\mathrm{F}}$ the many novel scientific devices which appeared during the War the hydrophone is in some ways the most remarkable. This is neither because it is an instrument of great complexity nor because any discovery of radical importance was in question, but rather because of the hold it obtained over popular imagination, and the new fields of speculation which were displayed to the less sophisticated, in stories of its use. We have become so accustomed to using our powers of hearing in our natural element that the very idea of sounds travelling in water was to many the revelation of a strange thing.

In a similar way, it appears to occasion less surprise when one ventures to estimate the distance of a cliff or a hillside by the simple expedient of shouting at it and using a stop-watch to determine the time of the echo, than when one states that the depth of water beneath a ship can be measured, even when the vessel is proceeding on its course, by an almost equally simple device. The object of this article is to give some account of the development, both in Great Britain and abroad, of what is now commonly known as the echo method of depth sounding, and to refer particularly to the type of apparatus which has been developed under the Admiralty, and to results which have been obtained with such apparatus, when used at sea under practical conditions.

The method of sounding by lead is probably almost as old as the art of navigation, and so old that history can help little in discovering its origin. Herodotus (circa 450 B.c.) not only refers to sounding by the lead, but he also mentions arming the lead with grease for obtaining samples of the sea-bed; so that we may say with certainty that for more than two thousand years - that is, until the invention of the Kelvin wire sounding-machine-this ancient method remained an unimproved and unchallenged guide to navigation. During the last few years publications received from the United States, France and Germany have shown that the new method, in which an acoustic impulse replaces the lead and wire, is under active development. We may therefore thank the War for giving us the hydrophone, and perhaps for removing some of the psychological difficulties which appear previously to have stood in the way of utilising underwater echoes.

In April I9I4 Prof. R. A. Fessenden experimented in America with a submarine signalling oscillator, which was made to emit a short note and then to receive the echo from the bottom. An observer provided with telephones and a stop-watch timed the interval between the original impulse and the echo as accurately as possible, but, since an error of r/ro of a second in the time recorded corresponds to an error of about 250 feet in depth, the results obtained must have been far from accurate. In May r9is, Marti, a Frenchman, experimented in the Channel with an apparatus in which the initial sound was produced by the detonation under water of a small charge of explosive. The outgoing and returning impulses were received with a hydrophone, and were recorded on a chronograph reading correctly to $\mathrm{I} / \mathrm{I}_{5} \mathrm{00}$ of a second,

1 Communication made by permission of the Admiralty by the staft of the Director of Scientific Research, Admiralty. corresponding to an error in depth of about I metre. Only 2 grams of explosive were necessary for depths up to 200 metres, and the system could be used with the vessel in motion up to speeds of ro knots. This method was proposed some time previously by Berggraf, a Norwegian inventor, but no reports of its use by him have been seen. More recently, Marti has used the impulse provided by a bullet fired into the water as a source of sound, while in the Behm system, which has been brought into a practical form in Germany, a detonator is fired out of a specially designed holder attached to the hull of the vessel. This apparatus has been made automatic and self-indicating, but it has the disadvantage that a very large supply of detonators is required to obtain anything like a complete line of soundings over a considerable distance.

In the foregoing paragraph we have considered methods which are based on the calculation of depth by the measurement of the time interval alone. Clearly, however, this is not the only possible method. Proposals have, for example, been made to determine depth by emitting definite quantities of energy and measuring the intensity of the received echo. The deeper the water the smaller will be the intensity of the returning sound. In theory it should be possible to calibrate the receiver so as to read depth of water in terms of intensity, but the accuracy of such a method would probably suffer on account of the variation in reflecting power of various types of sea bottom. We should expect a smaller quantity of energy to be reflected by a muddy or sandy bottom than by a clean smooth rock formation, even if the depth of water were the same in all cases.

In America, Hayes has developed a method which is especially suitable for use in shallow water. In his arrangement a transmitter is placed below water at one end of the ship, say well forward, while aft there is fitted an acoustic receiver of special type, by which the direction of travel of the received sound may be ascertained. The course of the sound from the transmitter to the receiver is assumed to be along the two sides of an isosceles triangle, of which the vertex is a point on the sea bottom, while the base is the distance between the transmitter and receiver. The angle which the direction of travel of the received sound makes with the line joining the receiver and transmitter having been determined, it is a simple matter to calculate the vertical height of the triangle, that is, the depth of water. This system is understood to have given good results in shallow water, but corrections must be introduced when the bottom is not horizontal, and a method of obtaining this correction has been elaborated. It has also been proposed to eliminate the transmitter and use the propeller noises instead. In this case the receiver would be fitted forward in the vessel.

In Great Britain attention has been paid chiefly to the development of a system depending on the measurement of the time interval, since the indications of an instrument working on this principle appear likely to be more useful under all conditions than those of any other system; and because it lends itself more easily to the production of a simple, robust, and NO. 2839 , VOL. I I 3$]$ 
trustworthy installation. An apparatus working on this principle has been designed by the Research Department of the Admiralty.

In this apparatus the source of sound is a steel diaphragm, about five inches in diameter, fixed to the hull of the ship. This diaphragm is set in vibration

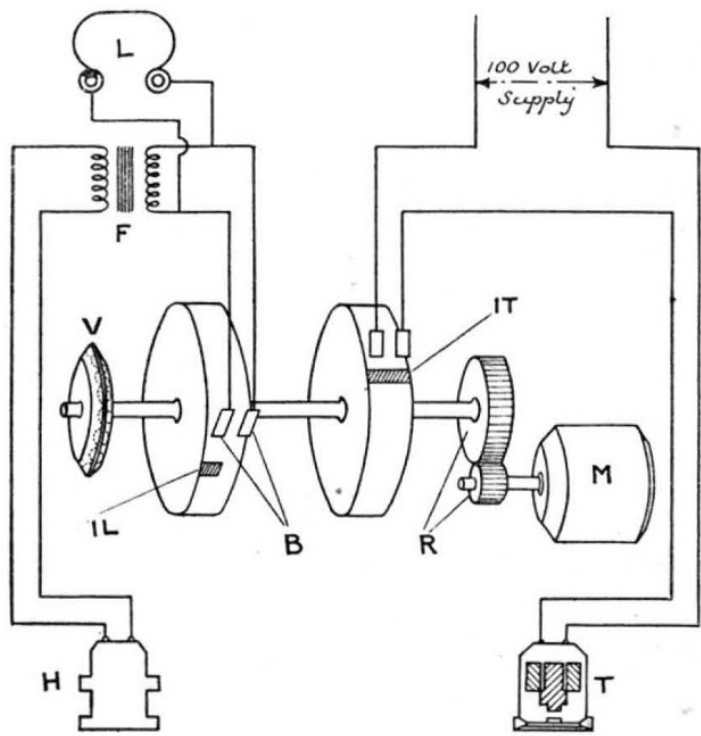

FIF. I.-Diagram of connexions: $T$, transmitter; IT, insulating segmen breaking transmitter supply; $M, \frac{1}{8}$ h.p. electric motor; $R$, 10 to I reduction gear ; $\mathrm{H}$, hydrophone ; $\mathrm{F}$, transformer; L, telephone; $\mathrm{IL}$, insulating segment breaking telephone circuit; $\mathrm{B}$, movable brushes in telephone circuit ; $v$, centrifugal governor.

at regular intervals by a small spring hammer which is withdrawn from the diaphragm by a solenoid, the circuit of the latter being broken automatically every half second. The note emitted by this transmitter has a frequency of $125^{\circ}$ per second, and when immersed in water the diaphragm is heavily damped, so that the
Installed in the vessel, and placed in any convenient position, is a r/8 h.p. electric motor running at I200 r.p.m., which drives two switches through a ro: I reduction gear. Constancy of speed within I per cent is obtained, in spite of large variations in the supply voltage, by the use of a specially designed centrifugal friction governor. One of the switches, which consists of a pair of brushes in contact with a rotating disc provided with an insulating segment, breaks the Io० volt D.C. supply to the transmitter every half-second. The other switch, running on the same shaft as this disc, short-circuits the telephones in the receiving circuit except as determined by the position, relative to the corresponding pair of brushes, of a second insulating segment.

When, therefore, the apparatus is running, no sound will be heard in the telephones unless the insulating segment in the telephone switch happens to open the telephone circuit at the instant when the transmitter is actuated, or at the instant when the echo returns from the bottom of the sea. By a simple mechanism, the position of the insulating segment in the telephone circuit can be displaced by hand relative to the corresponding pair of brushes, so that an interval of time, proportional to the angular displacement of the brushes, intervenes between the emission of an impulse by the transmitter and the opening of the telephone circuit. If, then, a sound is heard in the telephones, we know that the angular displacement of the brushes gives a measure, in terms of the known speed of rotation of the switch, of the time taken by the sound to travel from the transmitter to the receiver-a distance which is approximately double the depth of the water. To take a concrete example, the average velocity, over a depth of 30 fathoms, of sound in sea-water of 35 per mille salinity and at a temperature of $15^{\circ} \mathrm{C}$. is $4935 \mathrm{ft}$. per second. Let us suppose that the angular displacement of the brushes relative to the insulating segment

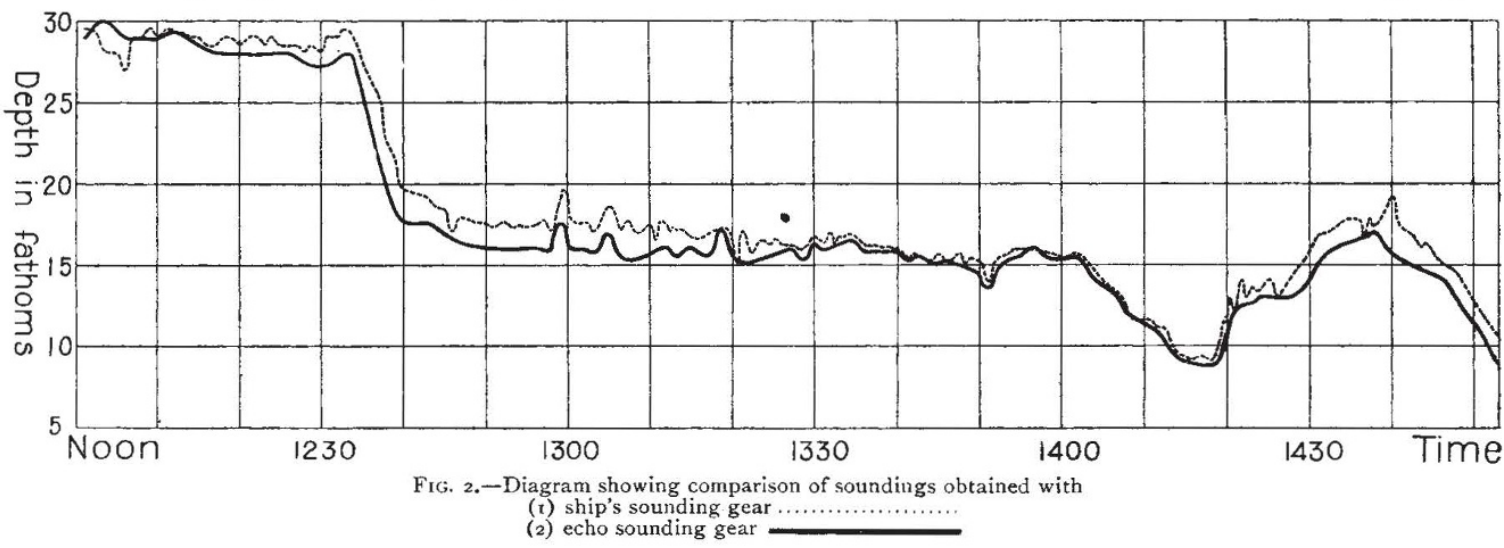

energy communicated to it by the hammer is quickly transferred to the water. The receiving hydrophone is a simple microphone enclosed in a rubber body immersed in the water. This type of hydrophone is comparatively aperiodic, and has been found suitable for the work ; since it is necessary when one is dealing with such small time intervals that both the transmitter and receiver should act quickly and not confuse the original sound and the echo by continuing to vibrate after an impulse. in the telephone circuit, when an echo is heard, is $18^{\circ}$. The time taken by the segment to rotate through $18^{\circ}$ is $18 / 360 \times I / 2$ second or $1 / 40$ second, since the segment goes round twice per second. In this time the sound will have travelled $123^{3}$ feet, and the depth of water is therefore a little over ten fathoms. The apparatus is shown diagrammatically in Fig. $\mathbf{r}$.

As soon as the apparatus was tried at sea, it was found that the system possessed a further important asset which one might describe as accidental, or at least 
unlooked for. One of the chief difficulties encountered in depth sounding with the vessel under way, using transmitters of moderate power, is due to the loud disturbances caused by movements of the water and sounds within the ship. If all these noises are listened to in the telephones without the short-circuiting device the echo may be difficult to distinguish, because it is not loud enough to be heard distinctly through the

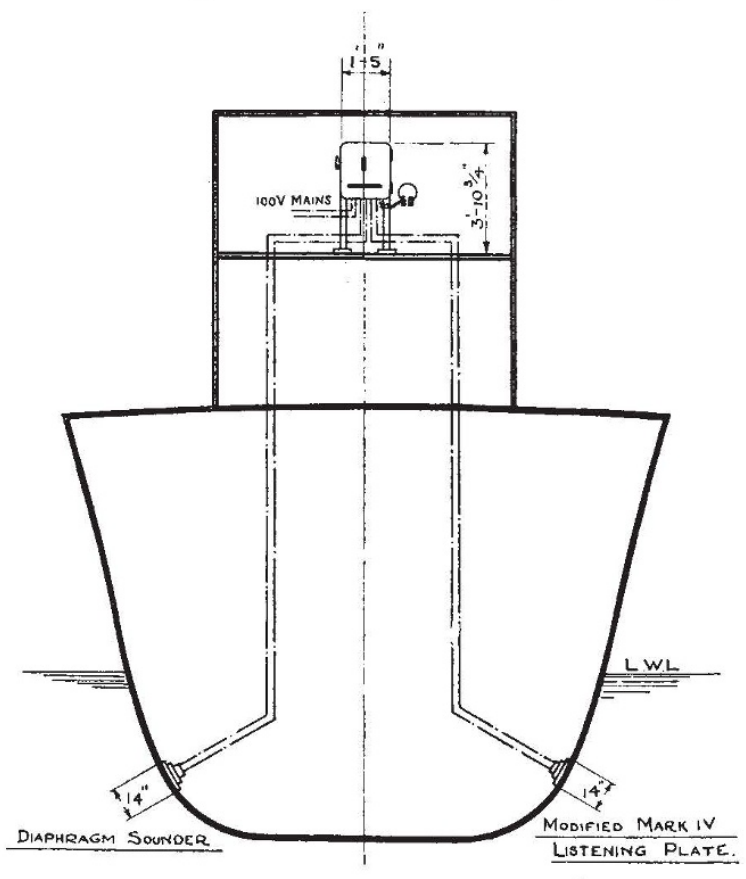

FIG. 3.-General arrangement of inboard apparatus.

other disturbances; but when the listening period is reduced to a small fraction of a second by the rotating switch, the echo is found to be clear and sharp.

In the specimen chart which is here reproduced (Fig. 2), Somerville wire and sonic soundings obtained on a trial run are compared. The apparatus was in this case handed over to the officers of an Admiralty survey vessel, who had had little or no prior experience of its use. The agreement is none the less good, and this may be taken as an indication of the simplicity and trustworthiness of the gear. In a report from the commanding officer of the vessel it is stated that the soundings were taken on a continuous run at a speed of six knots, under conditions which were by no means favourable - the send of the sea being sufficient to account for the four feet difference between the echo sounding and that obtained with the Somerville gear. From other trials the conclusion has been drawn that trustworthy indications can be obtained in all depths from almost the shallowest water in which a vessel will float, up to 35 fathoms. This does not, however, represent the greatest depth which the apparatus can be expected to measure.

In the trial run to which we have referred above the installation was exactly as described, except that the instrument was graduated to read fathoms directly, instead of time, while the transmitter, instead of being fixed in the hull, was hung overboard and submerged three feet. The receiver was placed in a ten-inch sluice valve, in order to shield it so far as possible from water currents and from the direct effect of the transmitter. In very shallow water a correction must be applied to allow for the distance apart of the transmitter and receiver, but this can be done very simply by an alteration in the scale of the fathom indicator. More powerful transmitters are being built so that the gear may be used in water of greater depth, and no difficulty is anticipated in obtaining indications over a range of 200 fathoms or more, with only minor modifications to the present apparatus. In equipment now in use both the transmitter and receiver are fitted directly on the hull on opposite sides of the vessel, as shown in Fig. 3, and the hull acts as a screen shielding the receiver from the transmitter. It is confidently anticipated that great depths may thus be sounded by a vessel proceeding at any ordinary speed. Special transmitters, which will be capable of dealing with oceanic depths, are under construction.

It is perhaps too soon to prophesy what will be the future of the sonic depth-sounding apparatus, and in what sphere it will find its most valuable application. There appears, however, to be good ground for anticipating that it may ultimately play an important part in the general practice of navigation.

\section{The Physical Society of London. \\ Jubilee Celebrations.}

$A \mathrm{~N}$ event of the first importance in the scientific $\mathrm{A}^{\mathrm{N}}$ world, the jubilee of the Physical Society, was marked by a series of celebration meetings held on March 20-2I at the Institution of Electrical Engineers, an exhibition of apparatus and demonstrations of great historical interest, and a banquet held at the Connaught Rooms on March 22. The labours of Prof. A. O. Rankine and those who helped him to organise the celebrations were rewarded by the spontaneous vigour which characterised the proceedings throughout their course. At the banquet Mr. F. E. Smith made the important suggestion that kindred societies which, like the Physical Society, do not possess a building of their own should unite in providing a common home in some central position.
On March 20, delegates were received from numerous learned societies of Great Britain and other countries (the foreign delegates including Profs. Fabry, Wien, S. J. M. Allen, Zeeman, and others whose names are well known), and addresses in a variety of languages were presented. The president of the Physical Society, in welcoming the delegates, commented on the contrast between the laboratory equipment which existed at the date of the Society's origin and that of the present day, and on the importance which the Society had always attached to demonstrations and apparatus. The number of fellows had grown from 99 to 600 , and was rapidly increasing: amongst the younger fellows must be some of the intellectual giants of the future. Later in the afternoon the Guthrie lecture was 